\title{
Efficacy of Skinfill plus filler in the management of facial aging: a multicenter, post-marketing clinical study
}

\author{
Antonella Savoia', Nicoletta Onori ${ }^{2}$ and Alfonso Baldi ${ }^{3^{*}}$
}

\begin{abstract}
Background: Injectable dermal fillers are commonly used by physicians in the treatment of the signs of aging. The most commonly used dermal filler is hyaluronic acid. Skinfill plus (SFP) belongs to the family of monophasic monodensified fillers. In this post-marketing clinical study, we have evaluated the efficacy of SFP for the treatment of facial aging.

Methods: The study enrolled 109 patients in three different centers that were treated with various SFP fillers to treat facial aging. Analyses of the cosmetic effects were performed by using the Wrinkle Severity Rating Scale (WSRS) and the Global Aesthetic Improvement Scale (GAIS).

Results: Statistical analysis showed a significant effect on facial aging for all the SFP fillers used at all time-points studied. Moreover, a significant correlation was found, by analyzing the grade of facial aging, calculated by using the WSRS or the lifestyle of the patients (smokers or non-smokers) in relation to the cosmetic effects of the treatment.

Conclusions: The study confirms the good performance and safety of SFP for a range of facial indications in routine clinical practice.
\end{abstract}

Keywords: Dermal filler, Facial aging, Hyaluronic acid

\section{Background}

In recent years, injectable dermal fillers have challenged the use of more invasive esthetic surgical procedures, emerging into the armamentarium of products used by physicians in the treatment of the signs of aging (Brandt \& Cazzaniga, 2008; Carruthers et al., 2009; Palm, 2014). The most commonly used dermal filler is hyaluronic acid (HA) (Lorenc et al., 2013). More than 1.6 million HA filler procedures were performed in 2014 in the USA, making it the second most commonly used nonsurgical esthetic procedure after botulinum toxin. HA is a glucosaminoglycan biopolymer composed of strands of repeating chains of D-glucuronic acid and N-acetyl-Dglucosamine (Lee et al., 2015). It is a normal component of the extracellular matrix in humans as well as many other species. HA is commonly found in the muscle, synovial fluid, skin, and vitreous body of the eye, in the

\footnotetext{
* Correspondence: alfonsobaldi@tiscali.it

${ }^{3}$ Department of Environmental, Biological and Pharmaceutical Sciences and Technologies, Università degli Studi della Campania "L. Vanvitelli", Via Vivaldi, 43, 81100 Caserta, Italy

Full list of author information is available at the end of the article
}

human body (Cheon et al., 2016). HA is extremely hydrophilic and can bind many times its own weight in water. These properties make it a lubricant and an important structural component of tissues. Moreover, HA, being highly biocompatible, displays a low incidence of antigenic adverse events (Lee et al., 2015). Nevertheless, in its natural state, HA shows reduced biomechanical properties as dermal fillers, because of its poor viscoelasticity and short half-life when injected into normal skin (Schante et al., 2011).

The first HA dermal fillers available on the market were biphasic fillers, made up by crosslinked particles suspended in a non-crosslinked HA matrix acting as a lubricant (Tran et al., 2014; Flynn et al., 2011). These products (e.g., Restylane ${ }^{\circ}$, Q-Med AB, Uppsala, Sweden, a wholly owned subsidiary of Galderma, Fort Worth, TX, USA; HA concentration $20 \mathrm{mg} / \mathrm{mL}$ ) were manufactured with nonanimal-stabilized hyaluronic acid $\left(\mathrm{NASHA}^{\circ}\right)$ technology (Verpaele \& Strand, 2006).

A large selection of HA dermal fillers have since been designed. In particular, monophasic monodensified fillers 
do not undergo "sizing," a common phenomenon that happens when using biphasic gels and that disrupts the gel (Bogdan Allemann \& Baumann, 2008). Indeed, they encompass a single phase of HA with a single density (Tran et al., 2014). Diverse families of monophasic monodensified fillers exist depending on the manufacturing technology; such is the case for Skinfill plus (SFP), which is the object of this article. In contrast to other monodensified fillers, SFP after crosslinkage is subjected to a micronization process. This process is carried out by injecting under pressure the material in a closed circuit in which it operates as a fluid (compressed air, superheated steam, inert gases) at a very high speed. The material particles, intimately mixed with the fluid, are dragged in the cycle. The continuous variations in speed and direction, due to the shape of the circuit, cause countless collisions among the particles themselves, which are thus subjected to repeated breaking actions. This process is called Coesix ${ }^{\oplus}$ and gives the extreme maneuverability and fluidity equivalent to the fillers which have the same concentration of HA and crosslinking agent. The final result is a gel with viscosity that is lower than that of other fillers used for the same indication. These properties provide a more homogeneous intradermal distribution of the material (Reinmüller, 2008). The SFP range of products offers different densities of HA (concentration ranging from 15 to $25 \mathrm{mg} / \mathrm{mL}$ ) to suit different purposes regarding softtissue augmentation and rejuvenation. The objective of this paper is to describe the clinical evidence regarding the performance, tolerability, and safety of the SFP dermal fillers for soft-tissue augmentation and rejuvenation.

\section{Methods}

\section{Skinfill}

Skinfill plus (Promoitalia Group, Italy) is a pyrogen-free, colorless, transparent, viscoelastic, cohesive, and monophasic gel obtained by bacterial bio-fermentation and generated through crosslink of hyaluronate diluted in a solution buffered with phosphate (Schante et al., 2011). Skinfill plus is subjected to a physical process called micronization or micro-grinding, by crushing to very minute dimensions in the order of micrometers. The product is sterile and it is contained in two syringes of $1 \mathrm{~mL}$ each equipped with a needle of 30 or 27 G. Three variants (Silver, Gold, Diamond), which vary depending on the viscosity of the product, have been used in this protocol. In detail, (a) Silver is indicated for the correction of periocular wrinkles and it has to be injected in the superficial derma, (b) Gold is injected in the middle derma, and (c) Diamond is equipped with greater density and, therefore, indicated for the re-harmonization of facial contours, for the increase of soft tissues, and for the treatment of the folds of the nose genus furrow.

\section{Patients}

The study was conducted in accordance with the ethical standards of Good Clinical Practice, and the applicable sections of the national medical device law. All patients provided written informed consent before enrollment in the study. All the HA fillers of the SFP range were CE marked and were used as per labeling, that is, defects related to aging such as wrinkles, loss of volume, and treatment of wrinkles around the lips.

The inclusion criteria of the study were represented by a severe-moderate photo-aging. Moreover, they had to be free of diseases that could interfere in cutaneous aging evaluation and none of the patients had undergone other medical-aesthetical treatments (botulinic toxin, laser for skin resurfacing, intradermic RF) for the entire duration of the follow-up.

Criteria of exclusion from the study were as follows: previously received permanent implants; any facial surgery or invasive procedures such as laser therapy, chemical peeling, dermabrasion, and botulinum toxin injection, or treatment with dermal fillers in the same anatomical regions within the previous 12 months before enrollment; dermatological problem such as cutaneous lesions and hypertrophic scars; systemic diseases such as diabetes mellitus and connective tissue diseases or immune system disorders; a positive history for allergies to HA or cosmetic fillers; pregnant or lactating patients; patients taking any medication that in the investigator's judgment would prohibit inclusion in the study.

A total of 109 patients, predominantly women, aged 32 to 66 years, seeking esthetic treatment in the face were recruited from the investigators' patient pool. In detail, 21 patients have been treated with SFP Silver, 44 patients with SFP Gold, and 44 patients with SFP Diamond. The procedures, as well as the evaluations, were performed in three different centers by three different doctors.

\section{Clinical evaluation}

The clinical results have been based on two reference scales. The Wrinkle Severity Rating Scale (WSRS) scale which evaluates the condition of the wrinkle and, therefore, the degree of aging. This scale has been useful for grouping patients and for the follow-up at 6 and 9 months. The Global Aesthetic Improvement Scale (GAIS) supplies an instrument useful for the interpretation of the cosmetic result immediately after the first session, during the touch-up, at 3,6, and 9 months. The WSRS and the GAIS are in turn supported by a photographic evaluation at 0,3 , 6 , and 9 months. Finally, treated patients have filled a selfevaluation test to define the level of satisfaction.

\section{Treatment procedure}

The area to be treated was first cooled down with the application of ice for $10 \mathrm{~min}$; then, local anesthesia was 
carried out with lidocaine topic at $2 \%$ an hour before the beginning of the filler procedure. Prior to treatment at the first visit (V1), patients' baseline characteristics were documented, and a severity assessment of the area to be treated was performed using the appropriate WSRSs. During this visit, the patients were treated in one or more areas of the face with the SFP HA filler(s) selected by the investigator according to their usual practice and patients' needs. The volume to be injected (up to a total of $2 \mathrm{~mL}$ ) and the injection techniques were at the investigator's discretion. At a follow-up visit 14 days postinjection (V2), further assessments were performed, including optional touch-up injections, if required, in which case the same product as the one injected at V1 was used. The V2 follow-up visit coincided with the routine patient follow-up performed in clinical practice, typically scheduled for $\sim 2$ weeks after the initial treatment. Successively, the patients were re-checked at three (V3) and 9 months (V4) after the first treatment, to complete the follow-up.

All evaluations at a given site were carried out by the same investigator. Performance was assessed at rest using the GAIS at V1 (immediately post-injection) and at V2. In case of a touch-up injection at V2, the evaluation was performed prior to injection(s). For bilateral treatment, each side was scored separately. During the period of follow-up, also the eventual adverse reactions have been evaluated. Adverse reactions were defined as signs and symptoms linked to the injection of stabilized hyaluronic acid and in particular the following: reactions of hypersensitivity, inflammation, pain, hematomas in the injection site, and itching sensation. The incidence of reactions in the injection site has been evaluated after the first treatment session and during the 9 months of follow-up. The most common reactions observed during the follow-up have been the following: dyschromia, pustular rash, papules, and nodules.

\section{Statistical analysis}

Descriptive analysis was made using median values and 95\% confidence interval (CI). The differences in the WSRSs and in the GAIS scores in the different timepoints of each group were performed using Wilcoxon's test for nonparametric dependent continuous variables. Spearman Rho correlation tests were performed to define correlation between different parameters. SPSS software (version 17.00, SPSS, Chicago, USA) was used for statistical analysis. A $p$ (two tailed) value of less than 0.05 was considered to indicate statistical significance.

\section{Results}

The treatment with the different fillers has caused minimum discomfort, without any post-treatment pain. All patients have returned to their daily accomplishments immediately after the treatment. In a small number of patients (10\% of patients), hematomas have occurred and areas of hyperemia associated with itchiness or pain in case of pressure after the injection disappeared in a few days, following the application of anti-edemigen creams. Tables 1, 2, and 3 illustrate all the results obtained in this study using the WSRS and the GAIS described in the "Methods" section. The use of Skinfill plus Silver was limited to the correction of perioral and periocular wrinkles. It has determined an excellent therapeutic success in patients already at 15 days after the treatment with a statistically significant difference in the GAIS scores $(p=0.0001)$. In panels e and f of Fig. 1 , an exemplificative case is shown. In the group of patients treated with Skinfill plus Gold, the injection was performed in the middle derma and resulted in an optimal cosmetic for the correction of facial wrinkles. The results were statistically significant starting from 15 days after the treatment $(p=0.0001)$. In panel $\mathrm{c}$ and $\mathrm{d}$ of Fig. 1, an exemplificative case is shown. The Skinfill plus Diamond is equipped with greater density and, therefore, was indicated for the re-harmonization of facial contours, for the increase of soft tissues and consequentially of the lips, and for the treatment of the folds of nose genus furrow. Its use has given good results with appreciable cosmetic effects, that were statistically significant already 15 days after the treatment $(p=0.0001)$. In panel a and $\mathrm{b}$ of Fig. 1, an exemplificative case is shown. To note, for all the filler used, the cosmetic effects were still visible and significantly different from time 0 to 9 months after the treatment $(p=0.0001)$. Interestingly, we performed also statistical analysis to define the correlation between the cosmetic effects still visible 9 months after the treatment and

Table 1 Wrinkle Severity Rating Scale (WSRS)

\begin{tabular}{lll}
\hline Score & Description & Number of patients at 15 days \\
\hline 5 & Extreme & 0 for Silver \\
& 0 for Diamond \\
& 0 for Gold \\
& 0 for Silver \\
& 0 for Diamond \\
& Severe & 0 for Gold \\
& & 1 for Silver \\
& 4 for Gold \\
& & 6 for Diamond \\
& & 9 for Silver \\
& & 15 for Gold \\
& Light & 17 for Diamond \\
& & 11 for Silver \\
& & 25 for Gold \\
& & 21 for Diamond
\end{tabular}


Table 2 Global Aesthetic Improvement Scale (GAIS); a total number of 109 patients were enrolled in the study

\begin{tabular}{|c|c|c|c|}
\hline Score & Degree & Description & Number of patients at 15 days \\
\hline \multirow[t]{3}{*}{1} & \multirow[t]{3}{*}{ Outstanding improvement } & \multirow[t]{3}{*}{ Excellent cosmetic result for the implant of the filler } & 11 for Silver \\
\hline & & & 21 for Diamond \\
\hline & & & 24 for Gold \\
\hline \multirow[t]{3}{*}{2} & \multirow[t]{3}{*}{ Very improved } & \multirow{3}{*}{$\begin{array}{l}\text { Marked improvement, but not completely excellent. A touch-up would } \\
\text { improve the results. }\end{array}$} & 9 for Silver \\
\hline & & & 17 for Diamond \\
\hline & & & 18 for Gold \\
\hline \multirow[t]{3}{*}{3} & \multirow[t]{3}{*}{ Improved condition } & \multirow{3}{*}{$\begin{array}{l}\text { An improvement compared to the initial condition but a touch-up is } \\
\text { recommended. }\end{array}$} & 1 for Silver \\
\hline & & & 6 for Diamond \\
\hline & & & 2 for Gold \\
\hline \multirow[t]{3}{*}{4} & \multirow[t]{3}{*}{ Unaltered condition } & \multirow[t]{3}{*}{ The condition seems to be the same as the initial one. } & 0 for Silver \\
\hline & & & 0 for Diamond \\
\hline & & & 0 for Gold \\
\hline \multirow[t]{3}{*}{5} & \multirow[t]{3}{*}{ Worsened condition } & \multirow[t]{3}{*}{ The appearance has worsened compared to the initial condition. } & 0 for Silver \\
\hline & & & 0 for Gold \\
\hline & & & 0 for Diamond \\
\hline
\end{tabular}

two different parameters: the grade of aging of the skin, as determined by the WSRS and the lifestyle of the patients (smokers or non-smokers). Indeed, we found a statistically significant inverse correlation, by using the Spearman correlation test for both parameters $(p=0.023$ and $p=0.024)$. Finally, the self-evaluation test filled by the patients 9 months after the treatment confirmed the long-lasting good cosmetic effect of these procedures: $90 \%$ of the patients were within levels 1 and 2 of the scale (see Table 3 )

\section{Discussion}

The performance of the filler is influenced by several rheological parameters such as the concentration of HA, the molecular weight, the crosslinking, and the elasticity and viscosity. The degree of crosslinking of a filler is instead related to its ability to retain water, and, then, to determine a post-treatment edema. It should also be considered that the physical-chemical properties (crosslinking, HA concentration, viscosity, etc.) also affect the stability and thus the longevity of the treatment, and, therefore, they involve medical assessments that take into account the specific patient and the specific problem to be treated. The Skinfill plus range ensures a variability of these parameters in order to select the most suitable characteristics for the treatment that will be run

Table 3 Self-evaluation test performed at the end of the treatment

\begin{tabular}{ll}
\hline Score 1 (Patient very satisfied) & 56 patients \\
Score 2 (Patient satisfied) & 44 patients \\
Score 3 (Patient not completely satisfied) & 9 patients \\
Score 4 (Patient not satisfied) & 0 patients \\
\hline
\end{tabular}

with a right balance between the physical and chemical characteristics of hyaluronic acid during the injection in order to minimize the occurrence of adverse events associated with it (Ginat \& Schatz, 2012; Carruthers et al., 2010).

Regarding the concentration of the filler, the literature documents various concentrations, generally in the range of 1.5-3\% (Brandt \& Cazzaniga, 2008; Carruthers et al., 2009; Palm, 2014). The different concentrations are used to adapt the filler to the specific intended use and to the anatomical area. Indeed, the filler used in this study, has been formulated in three different concentrations to ensure a variable approach to different clinical conditions, as described in the "Methods" section. In fact, the different areas of the face vary in their underlying structures, requiring different product characteristics, injection volumes, and injection techniques.

This study enrolled 109 subjects who were predominantly women aged 32 to 66 years with severe-moderate photo-aging. The objectives of the study were to assess the safety and efficacy of Skinfill plus fillers in achieving significant correction in facial signs of aging and to assess whether these positive results obtained would persist over the 9-month duration of the study. This project was a multicenter, observational study in a clinical setting using three different crosslinked HA fillers of the Skinfill plus range. The choice of filler or their combination was based on the parameters defined in the "Methods" section. The study confirms the good performance and safety of SFP for a range of facial indications in routine clinical practice. The majority of patients showed an improvement on GAIS immediately after injection and after 9 months of observation posttreatment, when compared with the baseline. The percentage of responders was high for all facial 

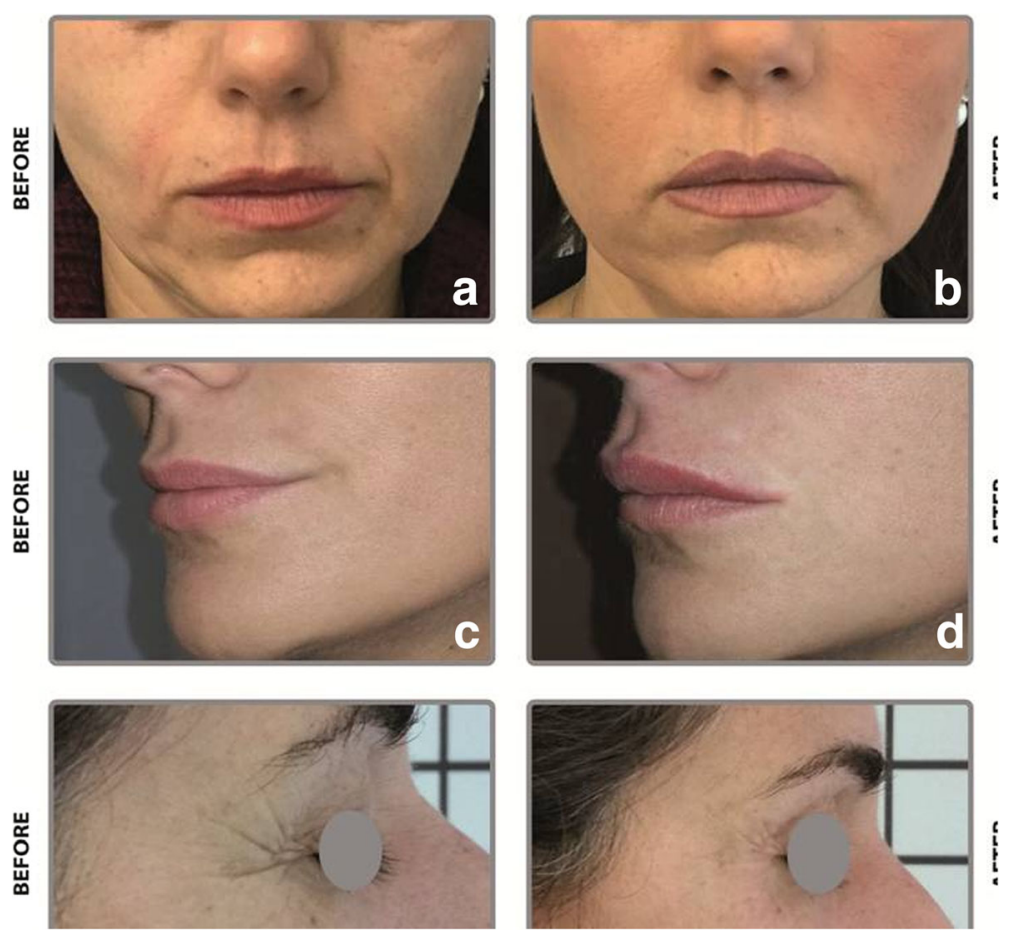

Fig. 1 Some examples of the results obtained with various Skinfill fillers on different body areas. a Patient at presentation with evident folds of nose genus furrow. $\mathbf{b}$ The same patients at 15 days of treatment with Skinfill Diamond. c Patient at presentation with wrinkles around lips. $\mathbf{d}$ The same patient at 6 months after treatment with SFP Gold. e Patient at presentation with periocular wrinkles. $\mathbf{f}$ The same patient at 9 months after treatment with Skinfill Silver

areas but was greatest for the lip area, followed by nasolabial folds.

Overall, patient satisfaction is a key parameter when evaluating the performance of dermal fillers, since esthetic interventions are optional and the patients play a decisive role in treatment choice. Our results, showing high patient satisfaction, corroborate the findings of several publications that show good patient outcomes, immediate results, and high satisfaction when using HA fillers for treatment of lines and wrinkles (Ginat \& Schatz, 2012; Carruthers et al., 2010) and for volume restoration (Muhn et al., 2012). Volumes of $<8 \mathrm{~mL}$ were sufficient for optimal esthetic outcomes and this can be explained by the fact that SFP has optimal rheological properties and a homogeneous pattern of tissue integration after intra- or subdermal implantation (Goh et al., 2014; Tran et al., 2014).

The data collected during this study also support the good safety profile of the SFP HA fillers. The minor adverse events reported, such as redness, swelling, and bruising, are to be expected with any type of dermal filler injection and are usually temporary. The most common post-injection adverse event was edema of the lips. This could occur depending on the product used, the injector's experience, the vessel supply in the treated area, the injection technique, and/ or the speed of injection. The literature shows that crosslinked hyaluronic acid fillers are considered safe and well tolerated, especially in cases where the HA is of bacterial origin (non-animal), and then characterized by a high biocompatibility (Savoia et al., 2011; Savoia et al., 2013).

Commonly observed adverse events are temporary, mild, and mainly related to the injection itself (burning, redness, itching, swelling, edema) (Brandt \& Cazzaniga, 2008; Carruthers et al., 2009; Palm, 2014). Despite the high safety profile of hyaluronic acid, the literature documents rare isolated cases of more serious adverse events and/or delays such as granulomas of the skin at a distance up to 2 years after the first injection, which is not found in the follow-up of patients treated with SFP (Ginat \& Schatz, 2012; Carruthers et al., 2010; Muhn et al., 2012).

\section{Conclusions}

In this context, the positive results and the good safety profile of the SFP HA fillers demonstrate the suitability of the fillers tested for comprehensive facial treatments in a normal clinical setting. Limitations of this study were the small number of patients in some of the treatment groups and the relatively short follow-up time. Moreover, it must be underlined the fact that GAIS and WSRS are commonly accepted scales to assess clinical efficacy of dermal fillers; nevertheless, they are essentially based on the subjective evaluation of the investigators and, consequently, 
can display significant bias. Considering also the fact that this was a single intervention cohort study, the results obtained, even if promising, must be confirmed and completed by further studies involving several cohorts of patients with a longer follow-up in order to better define the real efficacy of SFP for facial rejuvenation.

\section{Abbreviations}

GAIS: Global Aesthetic Improvement Scale; HA: Hyaluronic acid; SFP: Skinfill plus; WSRS: Wrinkle Severity Rating Scale

\section{Acknowledgements}

None

\section{Funding}

This research was partially supported by Promoitalia Group S.p.A.

\section{Availability of data and materials}

All data generated or analyzed during this study are included in this published article.

\section{Authors' contributions}

AS performed some of the treatments and collected the data from the other centers; NO recovered the data and performed statistical analyses; AS together with $A B$ conceived the work, analyzed the data, and wrote the paper. All authors read and approved the final manuscript.

\section{Ethics approval and consent to participate}

Research involving human material has been performed in accordance with the Declaration of Helsinki of 1975, as revised in 2000 and 2008; the ethical standards of Good Clinical Practice; the applicable sections of the national medical device law; and the ethical code of the Università degli Studi della Campania "L. Vanvitelli" (approved with D.R. 992/2012). An informed consent to participate in the study has been obtained from participants.

\section{Consent for publication}

A written informed consent for the publication of data and images relating

to individual participants has been obtained from the participants.

\section{Competing interests}

$A S$ and $A B$ are scientific advisors of Promoitalia Group S.p.A.

\section{Publisher's Note}

Springer Nature remains neutral with regard to jurisdictional claims in published maps and institutional affiliations.

\footnotetext{
Author details

${ }^{1}$ Buon Consiglio Hospital, Naples, Italy. ${ }^{2}$ San Giovanni Addolorata Hospital, Rome, Italy. ${ }^{3}$ Department of Environmental, Biological and Pharmaceutical Sciences and Technologies, Università degli Studi della Campania "L. Vanvitelli", Via Vivaldi, 43, 81100 Caserta, Italy.
}

Received: 14 September 2017 Accepted: 19 December 2017 Published online: 10 March 2018

\section{References}

Bogdan Allemann I, Baumann L. Hyaluronic acid gel (Juvederm) preparations in the treatment of facial wrinkles and folds. Clin Interv Aging. 2008;3:629-34.

Brandt FS, Cazzaniga A. Hyaluronic acid gel fillers in the management of facial aging. Clin Interv Aging. 2008;3:153-9.

Carruthers J, Cohen SR, Joseph JH, Narins RS, Rubin M. The science and art of dermal fillers for soft-tissue augmentation. J Drugs Dermatol. 2009:8:335-50.

Carruthers A, Carruthers J, Monheit GD, Davis PG, Tardie G. Multicenter, randomized, parallel- group study of the safety and effectiveness of onabotulinumtoxinA and hyaluronic acid dermal fillers (24-mg/ml smooth, cohesive gel) alone and in combination for lower facial rejuvenation. Dermatol Surg. 2010;36:2121-34
Cheon C, Kim Y, Son S, Lee DY, Kim J, Kwon M, Kim Y, Kim S. Viscoelasticity of hyaluronic acid dermal fillers prepared by crosslinked HA microspheres. Polym. Korea. 2016:40:600-6.

Flynn TC, Sarazin D, Bezzola A, Terrani C, Micheels P. Comparative histology of intradermal implantation of mono and biphasic hyaluronic acid fillers. Dermatol Surg. 2011;37:637-43.

Ginat DT, Schatz CJ. Imaging Features of Midface Injectable Fillers and Associated Complications. AJNR Am J Neuroradiol PubMed PMID: 22837310 (2012)

Goh AS, Kohn JC, Rootman DB, Lin JL, Goldberg RA. Hyaluronic acid gel distribution pattern in periocular area with high-resolution ultrasound imaging. Aesthet Surg J. 2014;34:510-5.

Lee DY, Cheon C, Son S, Kim Y, Kim J, Jang J, Kim S. Influence of molecular weight on swelling and elastic behavior of hyaluronic acid dermal fillers. Polym Korea. 2015;39:976-80.

Lorenc ZP, Fagien S, Flynn TC, Waldorf HA. Review of key Belotero Balance safety and efficacy trials. Plast Reconstr Surg. 2013;132:33S-40S.

Muhn C, Rosen N, Solish N, et al. The evolving role of hyaluronic acid fillers for facial volume restoration and contouring: a Canadian overview. Clin Cosmet Investig Dermatol. 2012;5:147-58.

Palm MD. Filler frontier: what's new and heading West to the US market. Semin Cutan Med Surg. 2014;33:157-63.

Reinmüller J. Hyaluronsäure in der ästhetischen Medizin - Historie, Entwicklung, heutige Bedeutung [Hyaluronic acid in aesthetics - History, development, and today's importance]. J Dtsch Dermatol Ges. 2008;6:54-9.

Savoia A, Vannini F, Baldi A. Radiofrequency waves with filling and peeling substances: An innovative minimally invasive technique for facial rejuvenation. Dermatol Ther (Heidelb). 2011;1:2-10.

Savoia A, Landi S, Baldi A. A new minimally invasive mesotherapy technique for facial rejuvenation. Dermatol Ther (Heidelb). 2013;3:83-93.

Schante CE, Zuber G, Herlin C, Vandamme TF. Chemical modifications of hyaluronic acid for the synthesis of derivatives for a broad range of biomedical applications. Carbohydr Polym. 2011;85:469-89.

Tran C, Carraux P, Micheels P, Kaya G, Salomon D. In vivo bio-integration of three hyaluronic acid fillers in human skin: a histological study. Dermatology. 2014; 228:47-54.

Verpaele A, Strand A. Restylane SubQ, a non-animal stabilized hyaluronic acid gel for soft tissue augmentation of the mid- and lower face. Aesthet Surg J. 2006:26:510-7
Submit your next manuscript to BioMed Central and we will help you at every step:

- We accept pre-submission inquiries

- Our selector tool helps you to find the most relevant journal

- We provide round the clock customer support

- Convenient online submission

- Thorough peer review

- Inclusion in PubMed and all major indexing services

- Maximum visibility for your research

Submit your manuscript at www.biomedcentral.com/submit
Biomed Central 